L'identificazione precoce di individui infetti a rischio elevato di progressione a TB attiva e di soggetti con malattia conclamata consente di ridurre le fonti di contagio nella popolazione. La diagnosi di ITBL si fonda tradizionalmente sull'impiego del test cutaneo tubercolinico secondo Mantoux (TCT), che presenta alcune limitazioni: specificità bassa (dovuta a crossreattività del derivato proteico purificato PPD utilizzato nel TCT con il vaccino BCG e i micobatteri ambientali); effetto "booster"; mancata standardizzazione nell'esecuzione e nella valutazione del test da parte dell'operatore; necessità di una visita di ritorno per l'interpretazione del risultato.

Il QuantiFERON ${ }^{\circledR}$-TB GOLD (QFT) è un test immunoenzimatico che valuta la risposta immune cellulo-mediata misurando la produzione di interferone-gamma da parte dei linfociti stimolati con antigeni specifici del Mycobacterium tuberculosis complex (ESAT-6,CFP-10,TB7.7(p4)).

Un prelievo di sangue intero è incubato in presenza e in assenza dei suddetti antigeni per $24 \mathrm{~h}$ a $37^{\circ} \mathrm{C}$ e il plasma recuperato può essere analizzato immediatamente o conservato $\left(4^{\circ} \mathrm{C}\right.$ per 4 settimane; $-20^{\circ} \mathrm{C}$ per 3 mesi).

Nel primo semestre del 2005 soggetti in regime di ricovero ed ambulatoriali, tra cui alcuni dializzati, con sospetta malattia tubercolare, familiari ed operatori sanitari venuti a contatto con pazienti con TB bacillifera sono stati esaminati con il QFT.

I risultati ottenuti sono stati confrontati, ove possibile, con il TCT. Sono state rilevate alcune discordanze.

Tra queste si segnala il caso di un operatore sanitario, analizzato pochi mesi dopo il contatto con un paziente con TB bacillifera, con TCT negativo e QFT positivo, che suggerisce una sensibilità maggiore del QFT almeno nel primo periodo dell'infezione.

Inoltre, sono stati riscontrati diversi casi con TCT positivo e QFT negativo attualmente oggetto di approfondimenti; alcuni di questi sono associati a vaccinazione con BCG.

\title{
INFEZIONE TUBERCOLARE:VALUTAZIONE DI UNTEST IMMUNOENZIMATICO BASATO SULLA RISPOSTA CELLULO-MEDIATA.
}

\author{
Cappiello G.'; Rivitti R. ${ }^{2}$; Longo R.';Visca M.'; Romano S.'; \\ Cava MC.'; Spanò A.' \\ U.O.C. Microbiologia, Virologia ed Immunologia, \\ ¿U.O.C. Pneumologia, \\ Ospedale "S. Pertini" - Roma
}

La diagnosi ed il trattamento dell'infezione tubercolare latente (ITBL) sono considerate priorità assolute per il controllo e l'eliminazione della tubercolosi (TB). 